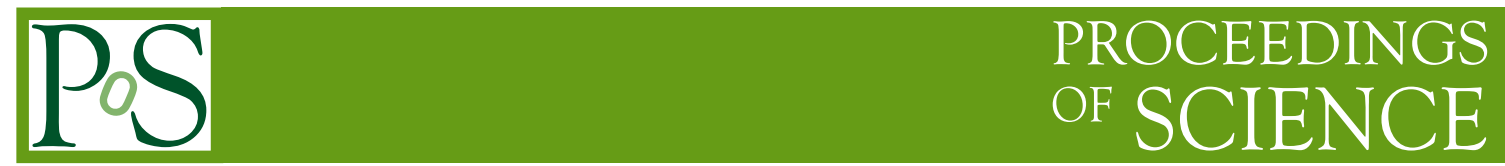

\title{
Searches for long-lived particles with the CMS detector
}

\section{Małgorzata KAZANA* for the CMS Collaboration}

National Centre for Nuclear Research, NCBJ, Pasteura 7, 02-093 Warsaw, Poland

E-mail: malgorzata.kazana@cern.ch

Selected CMS results of searches for exotic long-lived particles will be presented as available in the summer of 2019.

7th Annual Conference on Large Hadron Collider Physics - LHCP2019

20-25 May, 2019

Puebla, Mexico

${ }^{*}$ Speaker. 


\section{Introduction}

In this overview, the effort of the CMS experiment [1] to search for long-lived particles (LLPs) is shown. Recently published results are derived from data delivered by the CERN LHC colliding protons at centre-of-mass energy $\sqrt{s}=13 \mathrm{TeV}$ during a Run 2 period in $2015-2018$. The CMS searches are focused on different signatures with which a LLP may appear in the detector. Selected analyses are presented as follows.

\section{Signatures of long-lived particles}

Exotic long-lived particles on the order of $100 \mathrm{GeV}$ are not present in the Standard Model (SM), but their existence is predicted by many extensions of the SM. The LLP phenomenology is rich and varied. At the CERN LHC, if LLP is produced it will move with a speed close to the speed of light and travel for a significant distance before its decay, resulting in varied signatures of displaced or disappearing objects (tracks, jets, leptons, photons or vertices) not like a typical supersymmetric particle, which is expected to decay immediately after its production in the collision. Long-lived particles may cross the detector with a delay with respect to time needed by SM particles and they may have a specific ionization, which can be measured. LLPs may also be stopped inside the detector to due to interactions with the detector material. To detect LLPs, non-standard detection and triggering techniques are required.

\subsection{Displaced vertices}

The signature of displaced vertices is searched in the data sample, which was recorded by the CMS experiment during 2015 and 2016, corresponds to an integrated luminosity of $38.5 \mathrm{fb}^{-1}$. This search [2] uses benchmark signal models in which LLPs are pair-produced and each decays into two or more quarks, leading to a signal with multiple jets and two displaced vertices composed of many tracks.

The signal is discriminated from the SM background using the distance between the two vertices measured in the $\mathrm{x}-\mathrm{y}$ plane, which is defined as $d_{V V}$. In signal events, the two LLPs are emitted approximately back-to-back, leading to large separations. Therefore, signal events have two well-separated vertices, while background events are dominated by events with only one displaced vertex, usually close to the beam axis. The distribution of $d_{V V}$ is fitted to extract the signal, using templates to represent the $d_{V V}$ distributions for signal and background. The signal $d_{V V}$ templates are taken directly from simulation, with a distinct template for each signal mass and lifetime. The background template is constructed from events in data that have exactly one vertex. The expected distributions for a signal and background are shown in Fig. 1.

The background is estimated from data from samples of events with one vertex with at least 5 tracks, while the signal region is defined for events with two vertices and at least 5 tracks. The final selection includes requirements of at least of 4 jets and a high cut on the scalar sum of the transverse momenta $p_{T}$ of all selected jets, $H_{T}>600 \mathrm{GeV}$. In the signal extraction procedure, the $d_{V V}$ distribution is broken into three bins: $0-0.4 \mathrm{~mm}, 0.4-0.7 \mathrm{~mm}$, and $0.7-40 \mathrm{~mm}$. The two bins with $d_{V V}>0.4 \mathrm{~mm}$ have low background. This division maximizes the signal significance 
for scenarios with intermediate and long lifetimes. One event with two well-separated high-trackmultiplicity vertices is observed for $d_{V V}<0.4 \mathrm{~mm}$. In the region with $d_{V V}>0.4 \mathrm{~mm}$, there are no observed events.

Upper limits are placed on models of R-parity violating supersymmetry (RPV) in which LLPs are neutralinos or gluinos decaying solely into multijet final states (as shown in Fig. 2) or top squarks decaying solely into dijet final states. For neutralino, gluino, or top squark masses between 800 and $2600 \mathrm{GeV}$ and mean proper decay lengths between 1 and $40 \mathrm{~mm}$, the analysis excludes cross sections above $0.3 \mathrm{fb}$ at $95 \%$ confidence level (CL). Gluino and top squark masses are excluded below 2200 and $1400 \mathrm{GeV}$, respectively, for mean proper decay lengths between 0.6 and $80 \mathrm{~mm}$. While the search specifically addresses two models of RPV, the results are relevant to other models in which LLPs decay to final states with multiple tracks.

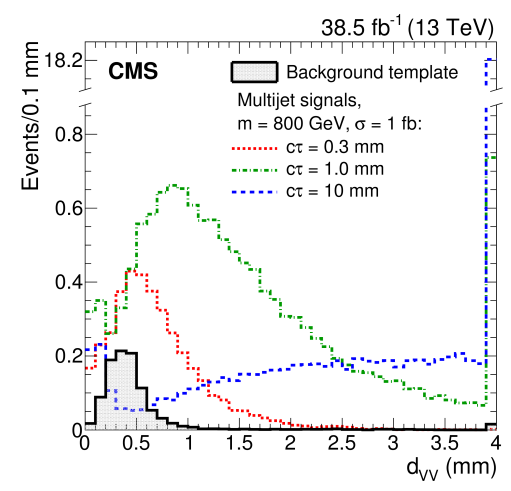

Figure 1: Distribution of the distance between vertices in the $\mathrm{x}-\mathrm{y}$ plane, $d_{V V}$, for simulated multijet signals with $m=800 \mathrm{GeV}$, production cross section $1 \mathrm{fb}^{-1}$, and $c \tau_{0}=0.3,1.0$, and $10 \mathrm{~mm}$, with the background template overlaid. All selection criteria are applied. The last bin includes the overflow events. Ref. [2]
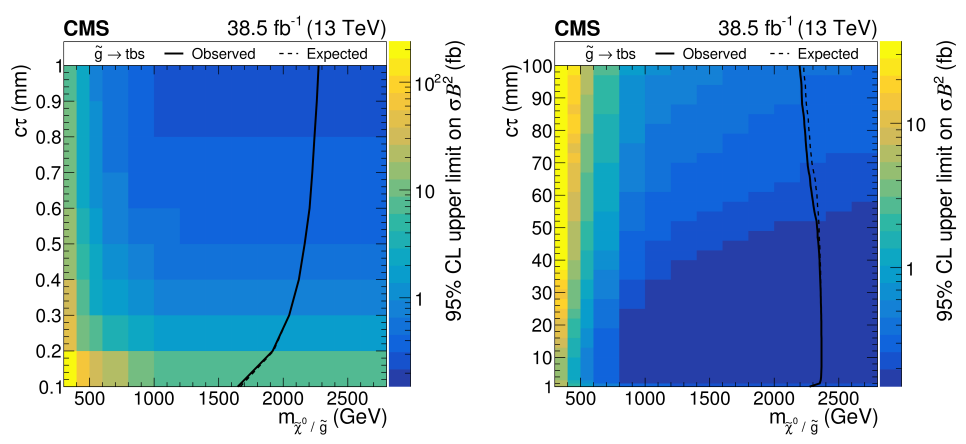

Figure 2: Observed $95 \%$ CL upper limits on on the cross-section $\times$ branching fraction $\left(\sigma B^{2}\right)$ for the multijet signal as a function of mass and mean proper decay length. The plot spans lifetimes $c \tau_{0}$ from 0.1 to $1 \mathrm{~mm}$ and 1 to $100 \mathrm{~mm}$, separately. The overlaid mass exclusion curves assumes gluino pair production cross sections and $100 \%$ branching fraction. Ref. [2]

\subsection{Displaced jets}

A search [3] for long-lived particles decaying into jets is presented. Data were collected with the CMS detector at the LHC from proton-proton collisions at a center-of-mass energy of $13 \mathrm{TeV}$ in 2016, corresponding to an integrated luminosity of $35.9 \mathrm{fb}^{-1}$. The search examines the distinctive topology of displaced tracks and secondary vertices.

The analysis examines dijets formed by jets clustered from energy deposits in the calorimeters. For the displaced-jet signal, the tracks left by charged particles originating from the decay of a longlived particle will usually exhibit large displacements with respect to the primary vertex, allowing the reconstruction of a secondary vertex within the associated dijet. The properties of the secondary vertex are utilized to discriminate between the long-lived signatures and the SM backgrounds. 


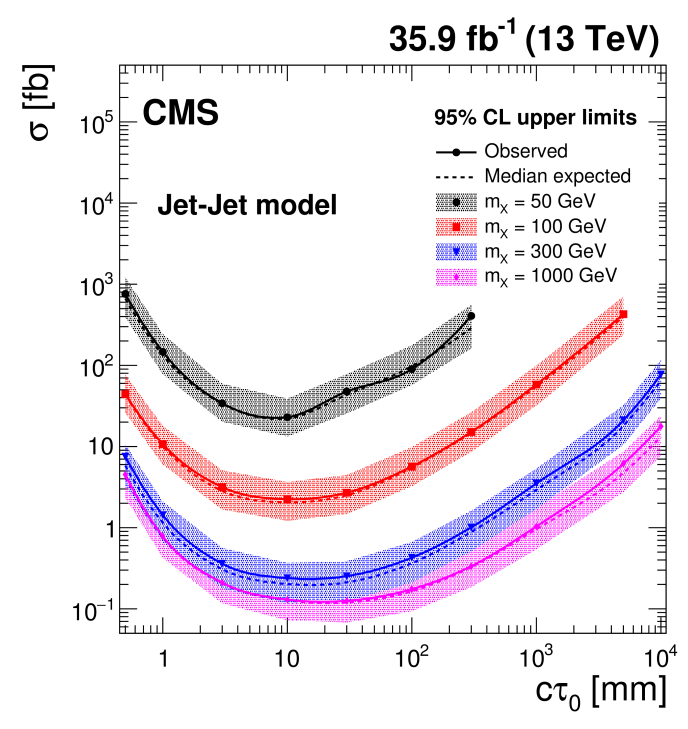

Figure 3: The expected and observed 95\% CL upper limits on the pair production cross section of the long-lived particle X, assuming a $100 \%$ branching fraction for $\mathrm{X}$ to decay to a quarkantiquark pair, shown at different particle $X$ masses and proper decay lengths for the Jet-Jet model. The solid (dashed) lines represent the observed (median expected) limits. The shaded bands represent the regions containing $68 \%$ of the distributions of the expected limits under the background-only hypothesis. Ref. [3]

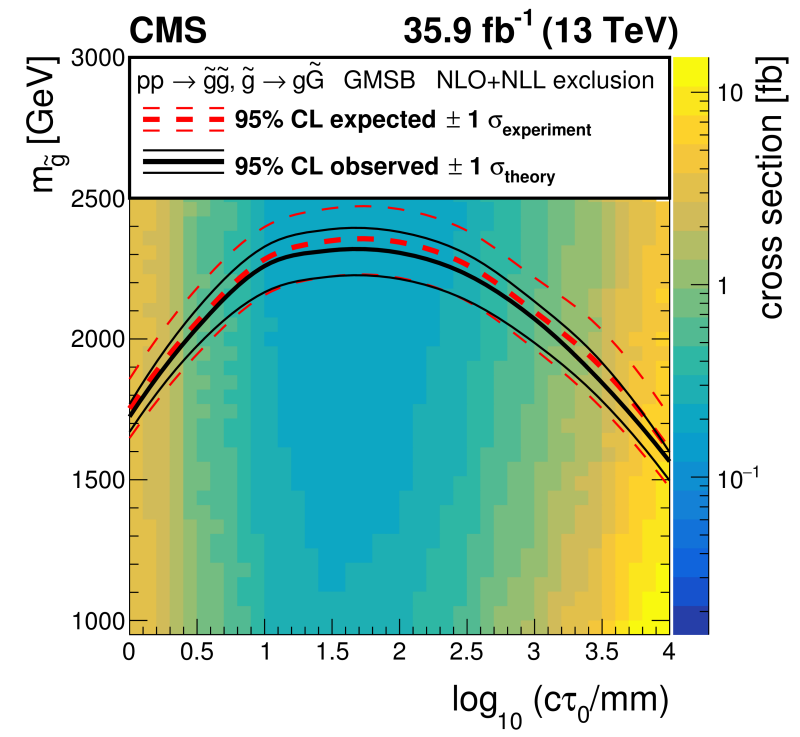

Figure 4: The expected and observed 95\% CL limits for the long-lived gluino model in the mass-lifetime plane, assuming a $100 \%$ branching fraction for $\tilde{g} \rightarrow$ $g \tilde{G}$ decays. The thick solid black (dashed red) line represents the observed (median expected) limits at $95 \% \mathrm{CL}$. The thin black lines represent the change in the observed limit due to the variation of the signal cross sections within their theoretical uncertainties. The thin red lines indicate the region containing $68 \%$ of the distribution of the expected limits under the background-only hypothesis. Ref. [3]

Although the objects studied in this analysis are dijets, two separate displaced single jets can pass the selection criteria, even when each displaced vertex contains only one jet.

The search is performed in 4 signal regions in bins of $H_{T}$ and the number of dijets passing the preselection criteria in order to gain sensitivity to long-lived particles with different masses. After applying all the selection criteria one event in the data is observed, while the total background prediction is of $1.03 \pm 0.19$ (stat) \pm 0.11 (syst) events. This observed event has an $H_{T}$ of $590 \mathrm{GeV}$ and yields a secondary vertex candidate, with a transverse decay length of $3.5 \mathrm{~cm}$ and a track multiplicity of 10. This is consistent with the presence of a bottom quark jet, where the bottom hadron travels in the tracker for an extremely long distance before it decays.

The selected events are found to be consistent with standard model predictions. Results for a simplified model in which long-lived neutral particles are pair produced and decay to two jets as shown in Fig. 3. Limits for pair production cross sections larger than $0.2 \mathrm{fb}$ are excluded at $95 \% \mathrm{CL}$ for a long-lived particle mass larger than $1000 \mathrm{GeV}$ and proper decay lengths between 3 and $130 \mathrm{~mm}$. Several supersymmetry models with gauge-mediated supersymmetry breaking (GMSB) or R-parity violation, where pair-produced long-lived gluinos or top squarks decay to several final-state topologies containing displaced jets, are also tested. For these models, in the 
mass ranges above $200 \mathrm{GeV}$, gluino masses up to $2300-2400 \mathrm{GeV}$ and top squark masses up to $1350-1600 \mathrm{GeV}$ are excluded for proper decay lengths approximately between 10 and $100 \mathrm{~mm}$. In particular, Fig. 4 shows results for the GMSB long-lived gluino model, where a branching fraction for $\tilde{g} \rightarrow g \tilde{G}$ decays is assumed to be $100 \%$. These gluinos are excluded for masses bellow $2300 \mathrm{GeV}$ and $c \tau_{0}$ between 20 and $110 \mathrm{~mm}$.

\subsection{Disappearing tracks}
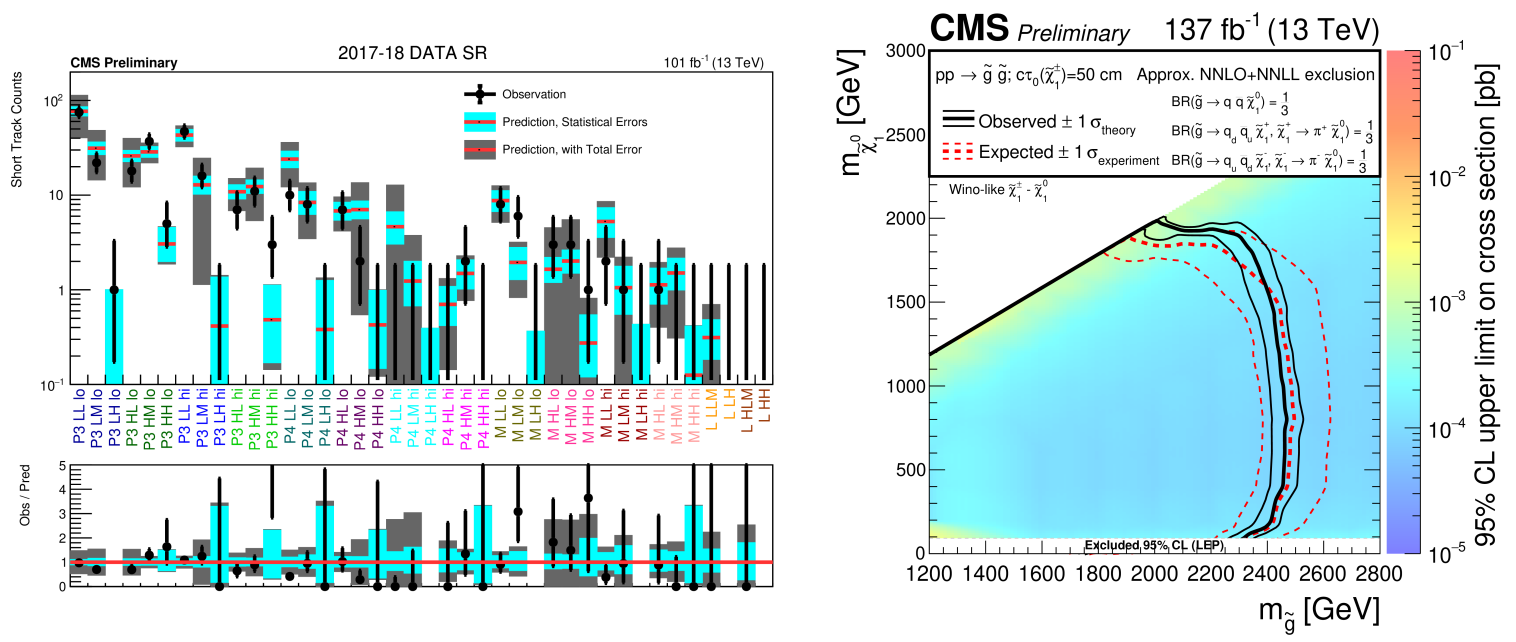

Figure 5: Comparison of estimated (pre-fit) background and observed data events in each of the 2017-2018 search regions, in the search for disappearing tracks. The red histogram represents the predicted background, while the black points are the actual observed data counts. The cyan band represents the statistical uncertainty on the prediction. The gray band represents the total uncertainty. The labels on the x-axes refers to regions of are explained in Ref [4]. Bins with no entry in the ratio have zero (pre-fit) predicted background. Ref. [4]

Figure 6: Exclusion limits at $95 \% \mathrm{CL}$ for gluino-mediated light-flavor $(\mathrm{u}, \mathrm{d}, \mathrm{s}, \mathrm{c})$ squark production with $c \tau_{0}\left(\tilde{\chi}_{1}^{ \pm}\right)=50 \mathrm{~cm}$. The area enclosed by the thick black curve represents the observed exclusion region, while the dashed red lines indicate the expected limits and their \pm 1 standard deviation ranges. The thin black lines show the effect of the theoretical uncertainties on the signal cross section. Ref. [4]

Searches [4] for new phenomena are performed using events with jets and significant transverse momentum imbalance. In events with at least two jets, the transverse momentum imbalance is inferred through the $M_{T 2}$ variable. The results are based on a sample of proton-proton collisions collected with the CMS detector during the LHC Run 2 (2016 - 2018) and correspond to an integrated luminosity of $137 \mathrm{fb}^{-1}$ taken at a center-of-mass energy of $13 \mathrm{TeV}$.

The search aims at extending the sensitivity of the standard $M_{T 2}$ analysis towards scenarios where the mass spectrum of potential new particles is compressed. In such scenarios, theories foresee the presence of new LLPs that can be identified as disappearing tracks when they decay within the volume of the tracking detector. A disappearing track is identified as a well reconstructed isolated track, disappearing within the volume of the CMS tracking detector, which forms a short track (ST). To ensure this, the presence of at least two outer layers of the tracking detector without a measurement is required. Because the track disappears within the tracking detector and it is 
not associated to any energy deposits in the calorimeters, this track is not accounted for in the calculation of a missing transverse energy (MET).

The search exploits the presence of disappearing tracks in order to suppress the background from SM processes, and to enhance the sensitivity towards these scenarios. The search uses events with at least two jets, and the $M_{T 2}$ variable to further suppress the background from SM processes. Event counts in bins of number of jets in the event $N_{j}$, the scalar sum of the transverse momenta $p_{T}$ of all selected jets $H_{T}$, disappearing track length, and $p_{T}$ of the short track. This results in 68 signal regions (SRs) for data of 2016 and 2017 - 2018 separately. The background estimates and corresponding uncertainties rely exclusively on the inputs from control samples and simulation, and are referred to as pre-fit background results. Fig. 5 shows a good agreement between the observation and the background prediction for 2017 - 2018. Similar results are obtained for 2016 data.

No excess event yield is observed above the predicted standard model background, leading to exclusion limits on pair-produced gluinos and squarks in simplified models of R-parity conserving supersymmetry. Mass limits as high as $2250 \mathrm{GeV}, 1770 \mathrm{GeV}, 1260 \mathrm{GeV}$ and $1225 \mathrm{GeV}$ are obtained from the inclusive standard $M_{T 2}$ search for gluinos, light-flavor squarks, bottom squarks and top squarks, respectively. The search for disappearing tracks extends the gluino mass limit to as much as $2460 \mathrm{GeV}$, as shown in Fig. 6 and the $\tilde{\chi}_{1}^{0}$ mass limit to as much as $2000 \mathrm{GeV}$, in models where the gluino can decay with equal probability to $\tilde{\chi}_{1}^{0}, \tilde{\chi}_{1}^{+}$, and $\tilde{\chi}_{1}^{-}$, and the $\tilde{\chi}_{1}^{ \pm}$are long-lived.

\subsection{Delayed jets}

A search [5] for long-lived particles decaying to displaced jets and missing transverse momentum represents the first application of a timing from the CMS electromagnetic calorimeter (ECAL) to a search for non-prompt jets from LLP decays. The data sample corresponds to an integrated luminosity of $137 \mathrm{fb}^{-1}$ of proton-proton collisions at a center-of-mass energy of $13 \mathrm{TeV}$ collected by the CMS experiment at the CERN LHC in 2016-2018.

Candidate signal events containing non-prompt jets are identified using the timing capabilities of the ECAL. The CMS ECAL consists of lead tungstate crystals, which provide coverage in pseudorapidity $|\eta|<1.48$ in a barrel region (EB) and $1.48<|\eta|<3.00$ in two endcap regions (EE). The ECAL measures the energy of incoming electromagnetic particles through the scintillation light produced in the lead tungstate crystals. Silicon avalanche photodiodes (APDs) are used as photodetectors in the barrel region. These are capable of measuring the time of incoming particles with a resolution as low as $200 \mathrm{ps}$ for energy deposits above $50 \mathrm{GeV}$. This analysis relies on the timing capabilities of the EB. Each ECAL crystal with an APD unit attached is referred to as an ECAL cell.

The jet timing is determined using all ECAL cells that satisfy reconstruction quality criteria. For each cell within the ECAL detector, the timing offset is defined such that a particle traveling at the speed of light from the center of the collision region to the cell position arrives at time zero. The time of the jet, $t_{j e t}$, is defined by the median cell time. The delay in the ECAL is expected to be a few ns for a $\mathrm{TeV}$ scale particle that travels about $1 \mathrm{~m}$ before decaying. With the jet timing requirement of $t_{j e t}>3 \mathrm{~ns}$, the dominant background sources are rejected, as shown in Fig. 7. The timing technique allows the reduction of background contributions to the few event level, while 
retaining high efficiency for signal signatures of one or more displaced jets and missing transverse energy in the final state.

There are three main background sources: beam halo muons deposits, out-of-time jets from core and satellite bunch collisions and jets originating from cosmic ray muons. The background sources are estimated from the control region using methods that rely on data. The estimated background yield in the control region (for MET $>300 \mathrm{GeV}$ and $t_{\text {jet }}>3 \mathrm{~ns}$ ) is $1.1_{-1.1}^{+2.5}$ events with dominant contribution from cosmic ray muons. This background prediction is consistent with the observation of 0 events. A representative GMSB model is used as a benchmark to quantify the

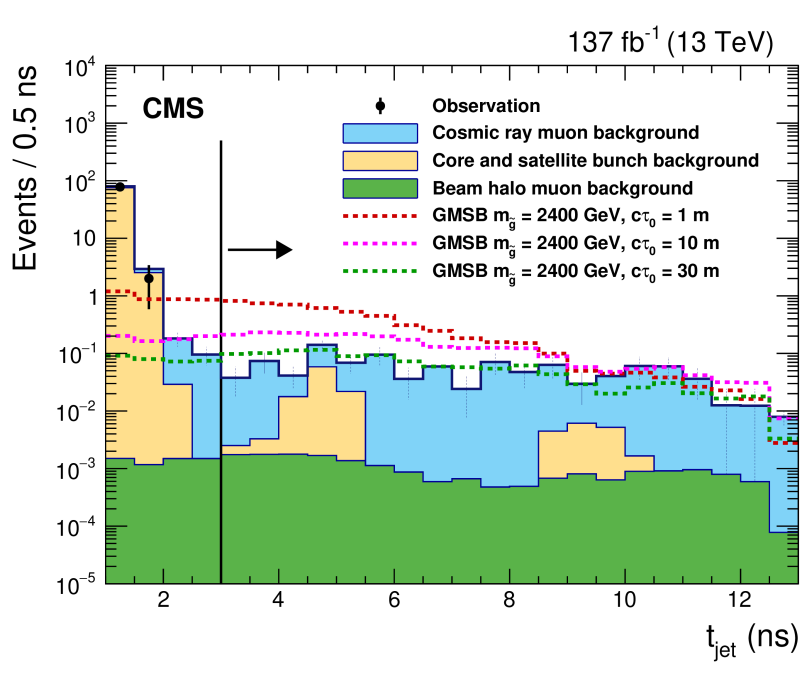

Figure 7: The timing distribution of the background sources predicted to contribute to the signal region, compared to those for a representative signal model. The time is defined by the jet in the event with the largest $t_{\text {jet }}$ passing the relevant selection. The distributions for the major background sources are taken from control regions and normalized to the predictions detailed in Section 6. The observed data are shown by the black points. No events are observed in data for $t_{\text {jet }}>3$ ns (indicated with a vertical black line). Ref. [5]

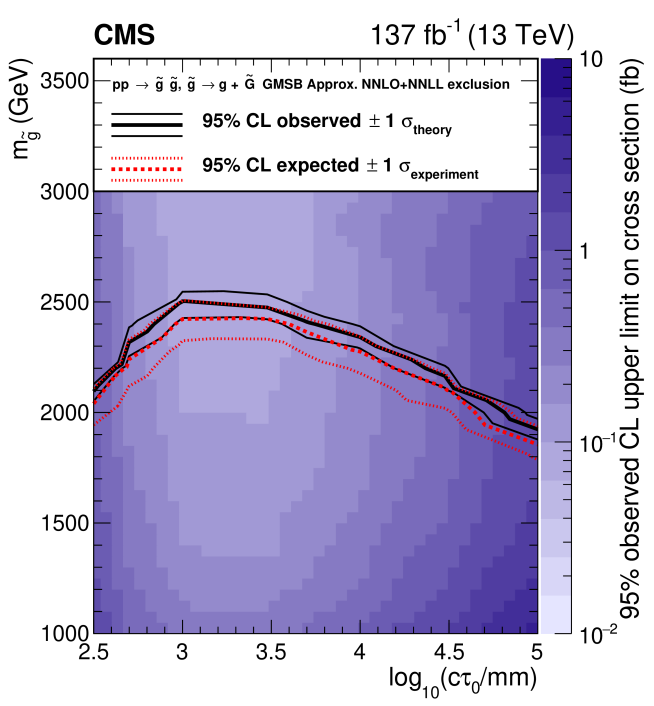

Figure 8: The observed upper limits at $95 \%$ $\mathrm{CL}$ for the gluino pair production cross section in the GMSB model, shown in the plane of $m_{\tilde{g}}$ and $c \tau_{0}$. A branching fraction of $100 \%$ for the gluino decay to a gluon and a gravitino is assumed. The area below the thick black curve represents the observed exclusion region, while the dashed red lines indicate the expected limits and their \pm 1 standard deviation ranges. The thin black lines show the effect of the theoretical uncertainties on the signal cross section. Ref. [5]

sensitivity of the search. In this model, pair-produced long-lived gluinos each decay into a gluon, which forms a jet, and a gravitino, which escapes the detector causing significant MET in the event. The dependence of the expected and observed upper limits as a function of a proper decay length $c \tau_{0}$ is shown in Fig. 8 for $m_{\tilde{g}}=2400 \mathrm{GeV}$. In this model, gluino masses up to 2100, 2500, and $1900 \mathrm{GeV}$ are excluded at $95 \%$ confidence level for proper decay lengths of $0.3,1$, and $100 \mathrm{~m}$, respectively. The observed limit in this analysis is compared to the results of the CMS displaced jet search [3], which is based on a data sample with integrated luminosity of $36.1 \mathrm{fb}$. The new limit shows the complementary coverage, for larger values of $c \tau_{0}$, which is illustrated in Fig. 9. These are the best limits to date for such massive gluinos with proper decay lengths greater than $\sim 0.5 \mathrm{~m}$. 


\subsection{Stopped particles}

A search [6] is presented for the decays of heavy exotic long-lived particles that are produced in proton-proton collisions at $\sqrt{s}=13 \mathrm{TeV}$ at the CERN LHC and come to rest in the CMS detector. Their decays would be visible during periods of time well separated from proton-proton collisions. Two decay scenarios of stopped LLPs are explored: a hadronic decay detected in the calorimeter and a decay into muons detected in the muon system. The calorimeter (muon) search covers a period of sensitivity totaling 721 (744) hours in $38.6(39.0) \mathrm{fb}^{-1}$ of data collected by the CMS detector in 2015 and 2016. Events are recorded out-of-time with collisions using a custom trigger. Since the background sources in both the calorimeter and the muon searches are not well modeled in simulation, the control samples in data are used to estimate their contributions after the full event selection is applied. For this purpose, dedicated cosmic run data were recorded during LHC machine technical stops, several days after collision runs. A negligible amount of long-lived signals produced during collisions could have decayed during these cosmic runs for the lifetimes considered in this analysis. The instrumental noise background estimate is extrapolated from the instrumental noise measured in these control samples. Most of the other sources of background are estimated from sideband regions of the main data sample, except for the cosmic ray muon background in the calorimeter search, which is estimated from MC simulation.

The results are interpreted in several scenarios that predict LLPs. Production cross section limits are set as a function of the mean proper lifetime and the mass of the LLPs, for lifetimes between $100 \mathrm{~ns}$ and 10 days as illustrated in Fig. 10. These are the most stringent limits to date on the mass of hadronically decaying stopped LLPs, and this is the first search at the LHC for stopped LLPs that decay to muons.

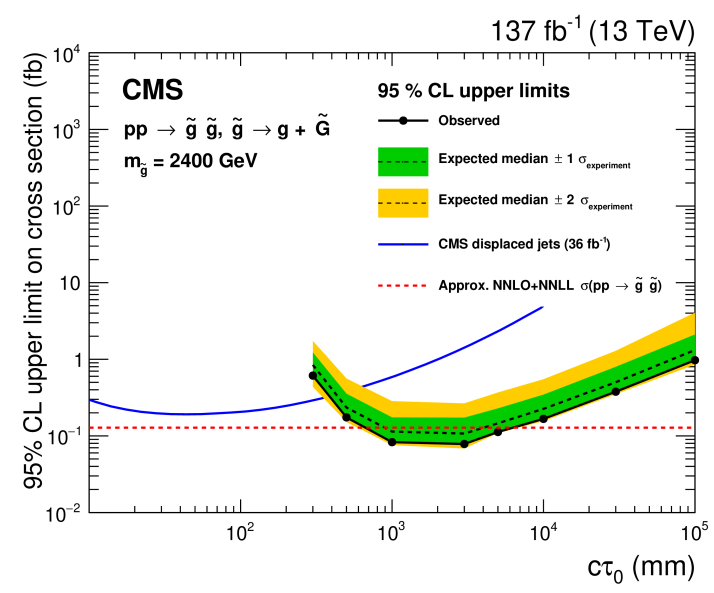

Figure 9: The observed and expected upper limits at $95 \% \mathrm{CL}$ on the gluino pair production cross section for a gluino GMSB model with $m_{\tilde{g}}=2400 \mathrm{GeV}$. The one (two) standard deviation variation in the expected limit is shown in the inner green (outer yellow) band. The blue solid line shows the observed limit obtained by the CMS displaced jet search [3]. Ref. [5]

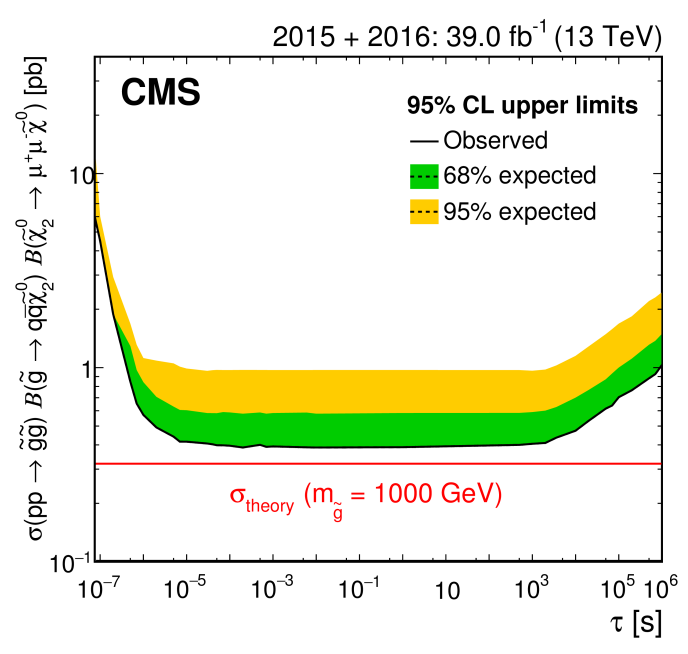

Figure 10: The $95 \% \mathrm{CL}$ upper limits on the cross-section $(\sigma) \times$ branching fraction $(B)$ for $1000 \mathrm{GeV}$ gluino pair production as a function of lifetime, for combined 2015 and 2016 data for the muon search. The theory lines assume $B=100 \%$. Ref. [6] 


\section{Summary and prospects}

The CMS experiment has a wide program for searching [7] for long-lived particles. The advanced techniques of reconstruction (displacement, timing and ionization) and triggering are used to improve sensitivity of the LLP searches. Since signatures of long-lived particles are unique, any detected signal of LLP would be a clear indication of new physics. Nevertheless, no LLP particle has been observed in the CMS detector with the analyzed LHC Run 2 data up to an integrated luminosity of $137 \mathrm{fb}^{-1}$. New exclusion limits on masses and cross sections have been set.

\section{Acknowledgments}

The author gives her acknowledgments to the conference committee for the invitation. This work is supported by the Polish National Science Centre (NCN) grant 2014/15/B/ST2/03998.

\section{References}

[1] CMS Collaboration, "The CMS experiment at the CERN LHC", JINST 3, S08004 (2008)

[2] CMS Collaboration, "Search for long-lived particles with displaced vertices in multijet events in proton-proton collisions at $\sqrt{s}=13 \mathrm{TeV}$ ". Phys. Rev. D 98 (2018) 092011, arXiv:1808.03078.

[3] CMS Collaboration, "Search for long-lived particles decaying into displaced jets in proton-proton collisions at $\sqrt{s}=13 \mathrm{TeV}$ ". Phys. Rev. D 99 (2019) 032011, arXiv:1811.07991.

[4] CMS Collaboration, "Searches for new phenomena in events with jets and high values of the MT2 variable, including signatures with disappearing tracks, in proton-proton collisions at $\sqrt{s}=13 \mathrm{TeV}$ ". CMS-PAS-SUS-19-005, arXiv:1909.03460, https: / / cds . cern. ch/record/2688695.

[5] CMS Collaboration, "Search for long-lived particles using nonprompt jets and missing transverse momentum with proton-proton collisions at $\sqrt{s}=13 \mathrm{TeV}$ ". Phys. Lett. B 797 (2019) 134876, Xiv:1906.06441.

[6] CMS Collaboration, "Search for decays of stopped exotic long-lived particles produced in proton-proton collisions at $\sqrt{s}=13 \mathrm{TeV}$ ". JHEP 05 (2018) 127, arXiv:1801.00359.

[7] https://twiki.cern.ch/twiki/bin/view/CMSPublic/PhysicsResultsEXO 